\title{
X-linked adrenal hypoplasia congenita and hypogonadotropic hypogonadism: Identification and in vitro study of a novel small indel in the NROB1 gene
}

\author{
TINGTING YU ${ }^{1,2}$, JIAN WANG $^{2}$, YONGGUO YU $^{3}$, XIAODONG HUANG $^{4}$, \\ QIHUA FU $^{5}$, YIPING SHEN ${ }^{5-7}$ and FUXIANG CHEN ${ }^{1}$
}

\begin{abstract}
${ }^{1}$ Department of Laboratory Medicine, Shanghai Ninth People's Hospital, Shanghai Jiaotong University School of Medicine, Shanghai 200011; ${ }^{2}$ Division of Birth Defects, Institute for Pediatric Translational Medicine, Shanghai Children's Medical Center, Shanghai Jiaotong University School of Medicine, Shanghai 200127; ${ }^{3}$ Department of Pediatric Endocrinology/Genetics,

Xinhua Hospital, Shanghai Jiao Tong University School of Medicine, Shanghai 200092;

Departments of ${ }^{4}$ Internal Medicine and ${ }^{5}$ Laboratory Medicine, Shanghai Children's Medical Center,

Shanghai Jiaotong University School of Medicine, Shanghai 200127, P.R. China; ${ }^{6}$ Department of Laboratory Medicine, Boston Children's Hospital, Boston, MA 02115; ${ }^{7}$ Claritas Genomics, Cambridge, MA 02139, USA
\end{abstract}

Received April 21, 2015; Accepted February 16, 2016

DOI: $10.3892 / \mathrm{mmr} .2016 .5006$

\begin{abstract}
DAX1 is an orphan nuclear receptor that has a key role in the development and function of the adrenal and reproductive axes. Mutations in $N R O B 1$, the gene encoding DAX1, result in X-linked adrenal hypoplasia congenita (AHC) and hypogonadotropic hypogonadism (HHG). A Chinese pedigree with $\mathrm{X}$-linked AHC and HHG was investigated in the present study. Sequence analysis identified a novel small indel variant, c.195_207delinsTG, in the NROB1 gene. To determine the effect of this variant on DAX1 expression, reverse-transcription quantitative PCR and western blot assays were performed. The mRNA expression levels in carriers of mutant NROB1 were significantly reduced (62\% decrease) compared to those in individuals with wild-type $N R O B I$ (WT). The c.195_207delinsTG mutation was demonstrated to lead to various truncated DAX1 proteins, including the C-terminal truncated DAX1, which was only detected in the cytoplasm, and the N-terminal truncated DAX1, which was present in the cytoplasm and nucleus. A luciferase assay was then performed to assess the repressor function of DAX1 in modulating steroidogenic factor 1 (SF-1)-mediated transactivation. WT DAX1 significantly suppressed the SF-1-mediated promoter activity of the steroidogenic acute regulatory protein
\end{abstract}

Correspondence to: Dr Fuxiang Chen, Department of Laboratory Medicine, Shanghai Ninth People's Hospital, Shanghai Jiaotong University School of Medicine, 639 Zhizaoju Road, Shanghai 200011, P.R. China

E-mail: chenfx@sjtu.edu.cn

Key words: adrenal hypoplasia congenital, hypogonadotropic hypogonadism, NROB1, DAX1, mutation by $35.5 \pm 1.9 \%$. In contrast to other known pathogenic mutations which abolish the repressor function of DAX1, the c.195_207delinsTG mutant proxkduced a higher repressor activity, demonstrating a $49.9 \pm 2.6 \%$ reduction of promoter activity. These findings suggested that the mutation of $N R O B 1$ in X-linked AHC with HHG enhanced the function of DAX1 to repress SF-1 activation, while DAX1 is expected to have additional roles in the pathological mechanism.

\section{Introduction}

$\mathrm{X}$-linked adrenal hypoplasia congenita (AHC) is a rare genetic disorder of adrenal gland development, characterized by absence or near absence of the permanent zone of the adrenal cortex (1). Patients with this condition usually present with primary adrenal failure, including salt-wasting, hyperpigmentation, failure to thrive, reduced serum cortisol and aldosterone and increased plasma adrenocorticotropic hormone (ACTH) (2). The majority of patients present with symptoms within the first two months of their life, whereas the remainder present later in childhood $(3,4)$. The condition is lethal if left untreated with appropriate steroid hormones (5). Besides adrenal insufficiency, hypogonadotropic hypogonadism (HHG) is a frequent feature of X-linked AHC. It is usually recognized during adolescence by the absence or interruption of normal pubertal development $(6,7)$.

$\mathrm{X}$-linked AHC was originally mapped to Xp21 and the nuclear receptor sub-family 0 , group $\mathrm{B}$, member 1 (NROB1) was subsequently identified by positional cloning as the gene responsible for X-linked AHC and HHG (2,8-11). The NROBI gene consists of two exons and encodes a 470-amino acid protein termed dosage-sensitive sex-reversal, adrenal hypoplasia congenital critical region on the X chromosome, protein 1 (DAX1) (12). DAX1 is an orphan member of the nuclear receptor superfamily. The DAX1 carboxy-terminal domain 
(CTD) is homologous to the ligand-binding domain (LBD) of other nuclear receptors, whereas the amino-terminal domain (NTD), which lacks the typical zinc finger DNA-binding motif, is composed of three short repeats, each containing an LXXLL motif (2). DAX1 is predominantly expressed in the adrenal cortex, gonads, hypothalamus and anterior pituitary $(13,14)$. Functional studies suggested that DAX1 is a repressor of gene transcription, acting in part by inhibiting the activity of another orphan nuclear receptor, steroidogenic factor 1 (SF-1), encoded by the NR5A1 gene (15-18). However, the exact role of DAX1 in the development and function of the adrenal and reproductive axes has remained elusive.

The present study described the clinical features of a Chinese male (age, 25 years) with X-linked AHC and HHG. A novel indel variant of the $N R O B I$ gene was identified and the functional effects of the mutation were assessed. The present study provided insight into the structure-function association of DAX1.

\section{Materials and methods}

Patient. A male patient (age, 25 years) was referred to the department with the main complaint of absence of pubertal development. He was full-term at birth, with a normal length and weight. His current height and weight were $167 \mathrm{~cm}$ and $55 \mathrm{~kg}$, respectively, which were below the $25^{\text {th }}$ percentile of the Chinese population. Physical examination revealed sparse pubic hair, small penis $(3 \mathrm{~cm})$ and low testicular volume $(3 \mathrm{ml}$ bilaterally) (Tanner stage 1) (19). Mild and diffuse skin pigmentation was also noticed, with a few hyperpigmented macules on the lips. Laboratory tests showed low concentrations of serum testosterone $(0.36 \mathrm{ng} / \mathrm{ml}$; normal range, $2.8-8.0 \mathrm{ng} / \mathrm{ml})$ and leutenizing hormone (1.16 IU/1; normal range, 1.7-8.6 IU/l). The serum follicle-stimulating hormone concentration was normal (7.16 IU/1; normal range, 1.5-12.4 IU/l). The patient had a past medical history of fatigue, nausea, hyperpigmentation and failure to thrive. The patient was diagnosed with Addison's disease at age 7 on the basis of a high serum ACTH concentration (299 pg/ml; normal range, up to $46 \mathrm{pg} / \mathrm{ml})$ in the presence of a low serum cortisol concentration $(2.5 \mathrm{ng} / \mathrm{ml}$; normal range, $5-25 \mathrm{ng} / \mathrm{ml}$ ). The patient had been treated with hydrocortisone since then. His current serum ACTH and cortisol concentrations $(43.69 \mathrm{pg} / \mathrm{ml}$ and $9.4 \mathrm{ng} / \mathrm{ml}$, respectively) were within the normal range. The patient was born to non-consanguineous parents, and while no other known case of X-linked AHC and HHG was present in the family pedigree (Fig. 1), the patient's mother was identified to be a carrier. The family members with the exception of the patient were healthy. They did not exhibit any hormone insufficiency disorders, such as adrenal insufficiency. The ethics committee of the Shanghai Children's Medical Center (Shanghai, China) approved the study and written informed consent was obtained from all subjects.

Molecular analysis of the NROB1 gene. Peripheral blood samples, to which ethylenediaminetetraacetic acid (BD, Franklin Lakes, NJ, USA) was added, were obtained from the patient, the patient's mother and 100 ethnicity-matched healthy individuals. Genomic DNA was isolated from the peripheral blood leukocytes using the QIAmp DNA Blood kit (Qiagen,
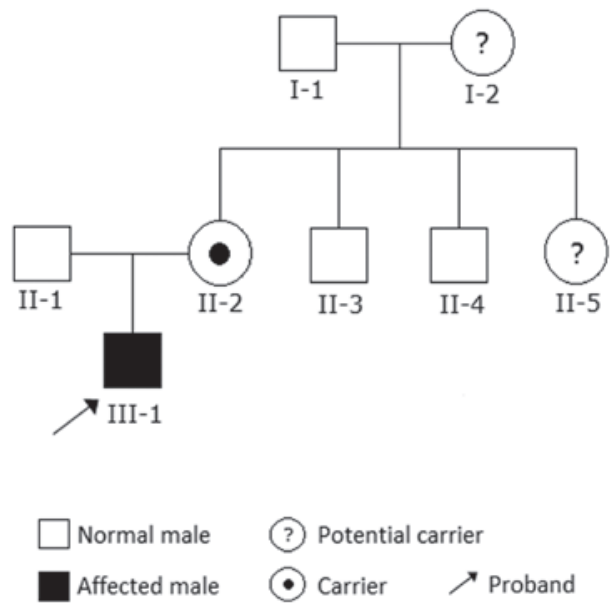

Figure 1. Pedigree of the Chinese family showing X-linked adrenal hypoplasia congenital with hypogonadotropic hypogonadism.

Hilden, Germany). All exons and exon-intron boundaries of the $N R O B 1$ gene from the proband's genomic DNA were amplified by polymerase chain reaction (PCR) using the primers listed in Table I as reported previously (20). The PCR products were analyzed by direct DNA sequencing on an ABI 3700 sequencer (Applied Biosystems; Thermo Fisher Scientific, Inc., Waltham, MA, USA). Only genomic fragments containing the mutation identified in the proband were amplified and sequenced for the proband's mother and the normal controls.

Plasmid construction and mutagenesis. The wild-type (WT) human NROBI and NR5Al complementary DNA (cDNA) clones were obtained from the Dana-Farber/Harvard Cancer Center DNA Resource Core (Boston, MA, USA). The NROBI cDNA was sub-cloned into pCSX/3Flag-DEST and pLenti6.3/V5-DEST Gateway vectors (Invitrogen; Thermo Fisher Scientific, Inc.) to generate the $\mathrm{N}$-terminal Flag-tagged $N R O B 1$ and $\mathrm{C}$-terminal V5-tagged NROBI expression vectors, respectively. The NR5A1 cDNA was sub-cloned into the pCSX/6HA-DEST Gateway vector. The NROBI c.195_207delinsTG and c.833T>C (Leu278Pro) mutations were created using a PCR-based $D p n \mathrm{I}$ treatment method (21). The mutagenic primers employed in the present study are listed in Table I. Steroidogenic acute regulatory protein (StAR) promoter sequences $(-1,096$ to +182$)$ from human genomic DNA were cloned into the pGL3-Enhancer vector (Promega Corp., Madison, WI, USA) to generate a luciferase reporter plasmid (StAR-luc). All of the final constructs were confirmed by DNA sequencing.

Cell culture and transient transfection. The HEK293T human embryonic kidney cell line (which does not express DAX1 protein) was obtained from the American Type Culture Collection (Manassas, VA, USA) and maintained in Dulbecco's modified Eagle's medium (DMEM; Thermo Fisher Scientific, Inc.) supplemented with $10 \%(\mathrm{v} / \mathrm{v})$ fetal bovine serum (FBS; Thermo Fisher Scientific, Inc.), $100 \mathrm{IU} / \mathrm{ml}$ penicillin and $100 \mu \mathrm{g} / \mathrm{ml}$ streptomycin (Sigma-Aldrich, St. Louis, MO, USA) in a humidified atmosphere with $5 \% \mathrm{CO}_{2}$ atmosphere at $37^{\circ} \mathrm{C}$. Transfection was performed using Lipofectamine 3000 (Thermo Fisher Scientific, Inc.) according to the manufacturer's instructions. 
Purification of total RNA and reverse-transcription quantitative PCR (RT-qPCR). HEK293T cells were collected $24 \mathrm{~h}$ after transfection. Total RNA was isolated using the RNeasy Mini Kit (Qiagen) and then reverse-transcribed into cDNA. SYBR green-based real-time qPCR was performed in a 96-well plate using FastStart Universal SYBR Green Master (Roche, Basel, Switzerland) in a StepOnePlus Real-Time PCR System (Invitrogen). The primers used for $\mathrm{qPCR}$ of $\mathrm{NROBI}$ are listed in Table I and were purchased from Beijing Genomics Institute (Shenzhen, China). The thermocycling protocol was: $95^{\circ} \mathrm{C}$ for $10 \mathrm{~min}$, followed by 40 cycles of $95^{\circ} \mathrm{C}$ for $15 \mathrm{sec}$ and $60^{\circ} \mathrm{C}$ for $30 \mathrm{sec}$. Values were normalized to $\beta$-actin using the $2^{-\Delta \Delta \mathrm{Ca}}$ method (22).

Western blot analysis. HEK293T cells were seeded $\left(5 \times 10^{5}\right.$ cells/well) into six-well plates and transfected with $\mathrm{N}$-terminal Flag-tagged and C-terminal V5-tagged NROBI expression vectors, respectively. Cytoplasmic and nuclear proteins were extracted using a nuclear and cytoplasmic extraction kit (Pierce Biotechnology, Inc., Rockford, IL, USA) at $24 \mathrm{~h}$ after transfection according to the manufacturer's instructions. The protein concentration was determined by the a Bicinchoninic Acid Protein Assay kit (Thermo Fisher Scientific, Inc.) according to the manufacturer's instructions and $25 \mu \mathrm{g}$ of cytoplasmic and nuclear proteins were loaded into each lane. Cytoplasmic and nuclear proteins were separated using $12 \%$ sodium dodecyl sulfate polyacrylamide gel electrophoresis Bio-Rad Laboratories, Inc. (Hercules, CA, USA), and transferred onto polyvinylidene difluoride membranes (Bio-Rad Laboratories, Inc.). The membranes were incubated with blocking solution for $1 \mathrm{~h}$ at room temperature under agitation. The blocking solution was Tris-buffered saline with Tween-20 (TBST) buffer containing 5\% non-fat milk. To make the $1 \mathrm{X}$ TBST buffer, $2.423 \mathrm{~g}$ Tris $\mathrm{Hcl}$ and $8.006 \mathrm{~g} \mathrm{NaCl}$ were dissolved in $800 \mathrm{ml}$ ultra pure water, and $1 \mathrm{ml}$ Tween-20 was added to adjust the $\mathrm{pH}$ to 7.6. Subsequently, ultra pure water was added to make the volume up to 1 litre. Tric $\mathrm{Hcl}, \mathrm{NaCl}$ and Tween-20 were obtained from Sigma-Aldrich. The membrane was probed with anti-Flag antibody (Sigma-Aldrich; cat. no. F3165; 1:1,000) or anti-V5 antibody (Abcam, Cambridge, MA, USA; cat. no. ab27671; 1:2,000), anti- $\beta$-tubulin (cat. no. T5201, Sigma-Aldrich; 1:2,000) and anti lamin B1 (Abcam; cat. no. ab133741; $1: 5,000)$ for $1 \mathrm{~h}$ at room temperature. $\beta$-tubulin and lamin B1 were used as cytoplasmic and nuclear loading controls, respectively. The membranes were then incubated with horseradish peroxidase (HRP)-conjugated goat-anti-mouse IgG (cat. no. ab97023, Abcam; 1:5,000) and HRP-conjugated goat-anti-rabbit IgG (cat. no. ab97051, Abcam; 1:5,000) for $1 \mathrm{~h}$ at room temperature. The membranes were washed 4 times in TBST buffer at room temperature under agitation, 5 min per wash, to remove residual primary and secondary antibodies. The blots were visualized using an enhanced chemiluminescnece Western Blotting Substrate (Thermo Fisher Scientific, Inc.). The imaging platform used was ImageQuant LAS 4000 mini (GE Healthcare, Pittsburgh, PA, USA).

Luciferase assay. The regulatory role of DAX1 in modulating transcription was monitored using the StAR-luc plasmid. In brief, HEK293T cells were seeded into 96-well plates at a density of $6 \times 10^{4}$ cells/well and incubated for $24 \mathrm{~h}$. The cells were co-transfected with $20 \mathrm{ng}$ StAR-luc, $0.5 \mathrm{ng}$ pRL-SV40, and $60 \mathrm{ng}$ WT or mutant DAX1, and treated with $20 \mathrm{ng}$ NR5A1 plasmid which encoded steroidogenic factor-1 (SF-1). Total DNA was kept constant by complementing each transfection with empty backbone plasmids whenever it was necessary. Luciferase activities were measured from cell lysates at $24 \mathrm{~h}$ post-transfection using the Dual-Glo luciferase assay system (Promega Corp.) and normalized to Renilla luciferase encoded by pRL-SV40. A reported NROB1 mutation, Leu278Pro, was used as the control (23).

Statistical analysis. Comparisons were made using the two-tailed Student's t-test. Statistical analysis was conducted using Microsoft Excel 2013 (Microsoft Corporation, Redmond, WA, USA). Values are expressed as the mean \pm standard deviation from three independent transient transfection assays. $\mathrm{P}<0.05$ was considered to indicate a statistically significant difference.

\section{Results}

Mutational analysis. Direct sequencing of the proband revealed a small indel variant in the $N R O B 1$ gene, which was maternally inherited (Fig. 2A). The indel variant, c.195_207delinsTG, was located in exon 1 of the $N R O B 1$ gene and was predicted to cause a frameshift at amino acid codon 66 (p.Cys66GlyfsX2, Fig. 2B) as predicted by the mutation analysis software named Alamut Visual v.2.7.1 (Interactive Biosoftware, Rouen, France). This variant has not been previously reported in the Human Genome Mutation Database (http://www.hgmd. cf.ac.uk) or control databases such as the 1,000 Genomes project (http://www.1000genomes.org), the National Heart, Lung and Blood Institute Gene Ontology Exome Sequencing Project (http://evs.gs.washington.edu/EVS) or the Exome Aggregation Consortium (http://exac.broadinstitute.org), and therefore represents a novel mutation, to the best of our knowledge. Furthermore, the mutation was not detected in the 100 ethnicity-matched healthy control subjects.

The c.195_207delinsTG mutation in the NROBI gene reduces $D A X 1 \mathrm{mRNA}$ expression in vitro. To investigate the effect of the c.195_207delinsTG mutation on NROB1 transcription, WT and c.195_207delinsTG mutant NROB1 expression vectors were transiently and individually transfected into HEK293T cells, followed by RNA extraction and RT-qPCR analysis. DAX1 mRNA expression in the NROB1 mutant was significantly reduced $(62 \%$ decrease; $\mathrm{P}<0.001)$ compared to that in the NROB1 WT group (Fig. 3).

The c.195_207delinsTG mutation in the NROB1 gene leads to low-level expression of truncated DAX1 protein. To investigate the effects of the c.195_207delinsTG mutation on the synthesis and sub-cellular localization of DAX1, N-terminal Flag-tagged and C-terminal V5-tagged NROB1 expression vectors were transiently and individually transfected into HEK293T cells, followed by extraction of cytoplasmic and nuclear proteins, which were subjected to western blot analysis. The WT DAX1 protein was $\sim 55 \mathrm{kDa}$ in length and localized to the nucleus as well as the cytoplasm (Fig. 4). Compared to the levels of the WT DAX1 protein, the levels of mutant DAX1 proteins 
Table I. Primers used for amplification and mutagenesis of NROBI.

\begin{tabular}{|c|c|c|}
\hline Primer & Sequence $\left(5^{\prime} \rightarrow 3^{\prime}\right)$ & Size (bp) \\
\hline NROB1-exon1.1-F & TCGAACCACCGAGGTCAT & 746 \\
\hline NROB1-exon1.1-R & CTGGTAGCGCCTCTTTACCC & \\
\hline NROB1-exon1.2-F & GCTTGCTCACTAGCTCAAAGC & 859 \\
\hline NROB1-exon1.2-R & TCACGATTTCTTCACCTTTGC & \\
\hline NROB1-exon2-F & TCTTGGACACGTTGCTTCTG & 717 \\
\hline NROB1-exon2-R & GCAGGTTCCATGAAATTGCT & \\
\hline 195_207delinsTG-F & GTGGCGCTCCTGTACCGTGGGTAAAGACCACCCACGG & 6558 \\
\hline 195_207delinsTG-R & CCGTGGGTGGTCTTTACCCACGGTACAGGAGCGCCAC & \\
\hline $833 \mathrm{~T}>\mathrm{C}-\mathrm{F}$ & CTGCTTCCAGGTGCCGCCCCTGGACCAG & 6569 \\
\hline $833 \mathrm{~T}>\mathrm{C}-\mathrm{R}$ & CTGGTCCAGGGGCGGCACCTGGAAGCAG & \\
\hline NROB1-qPCR-F & AGGGGACCGTGCTCTTTAAC & 214 \\
\hline NROBI-qPCR-R & ATGATGGGCCTGAAGAACAG & \\
\hline$\beta$-actin-F & GCCGGGACCTGACTGACTAC & 100 \\
\hline$\beta$-actin-R & TTCTCCTTAATGTCACGCACGAT & \\
\hline
\end{tabular}

F, forward; R, reverse; qPCR, quantitative polymerase chain reaction.

in the transfected cells were markedly lower. Application of the anti-Flag antibody showed that the c.195_207delinsTG mutation encoded a truncated protein $(\sim 15 \mathrm{kDa})$, which was predominantly located to the cytoplasm (Fig. 4A). Blotting with the anti-V5 antibody showed that the c.195_207delinsTG mutation encoded two additional truncated proteins $(\sim 50 \mathrm{kDa})$ that were contained in the nucleus as well as the cytoplasm (Fig. 4B).

The c.195_207delinsTG mutant of DAX1 shows enhanced suppression of SF-1-mediated StAR promoter activity. DAX1 has been previously shown to inhibit SF-1-mediated transactivation $(16,17)$. Therefore, a luciferase assay was performed in the present study to investigate whether the c.195_207delinsTG mutation impaired the repressor function of DAX1. As expected, WT DAX1 significantly suppressed SF-1-mediated StAR promoter activity by $35.5 \pm 1.9 \%$, whereas the 'classic' AHC mutant, Leu278Pro, showed a complete lack of repressive function. Compared to WT DAX1, the c.195_207delinsTG mutant of DAX1 showed an even higher repressive function, suppressing SF-1-induced StAR expression by $49.9 \pm 2.6 \%$ (Fig. 5 ).

\section{Discussion}

X-linked AHC with HHG is a rare genetic disorder caused by mutations in the NROBl gene. A Chinese pedigree was investigated in the present study. The proband, a 25-year-old male, presented with adrenal insufficiency during childhood and lack of development of secondary sexual characteristics during puberty. Molecular analysis revealed a small indel mutation in the NROBl gene. The diagnosis of X-linked AHC with HHG was established based on clinical presentation, laboratory tests and molecular analysis.

To date, $>200$ types of mutations of the NROBI gene have been recorded in the Human Gene Mutation Database.
The majority of the mutations reported are small deletions, followed by nonsense mutations and missense mutations. More than half of the mutations are clustered at the carboxyl terminus. To date, five small indel mutations in the NROB1 gene, including c.273_274delinsT, c.585delinsCC, c.986_987delinsA, c.1130delinsGT, and c.1376_1377delinsG have been described $(6,11,24-26)$. These small indel mutations lead to a frameshift and cause pre-mature truncation of the DAX1 protein. The present study reported on a novel small indel mutation in the NROB1 gene, c.195_207delinsTG, which affects the amino terminus of the DAX1 protein. Several adjacent mutations, including c.159delG, c.192C $>$ A, c.197dupG, c.226C $>\mathrm{T}$ and c. $243 \mathrm{C}>\mathrm{G}$, have been reported to cause X-linked AHC (27-31).

The NROB1 c.195_207delinsTG mutation is predicted to cause frameshift and pre-mature termination. To determine the effects of the c.195_207delinsTG mutation on DAX1 protein synthesis, transient expression studies were performed in HEK293T cells transfected with vectors carrying WT or mutant DAX1 cDNA. RT-qPCR analysis showed that the levels of the transcript of the NROB1 c.195_207delinsTG mutant were significantly reduced compared to those of the WT NROBl gene, indicating that the aberrant NROB1 mRNA with a pre-mature termination codon (PTC) may trigger nonsense-mediated mRNA decay (NMD). NMD is a well-known translation-coupled quality control system that recognizes and leads to a reduction in PTC-harboring mRNAs (32).

The decrease in DAX1 expression was also verified using a western blot assay. Similar to the findings of other studies $(20,33)$, the WT DAX1 protein was about $55 \mathrm{kDa}$ in size and localized in the nucleus as well as the cytoplasm. However, the NROB1 c.195_207delinsTG mutation resulted in various truncated DAX1 proteins. Western blot analysis using antibodies specific for the N-terminal Flag-tag revealed the presence of a truncated protein of $\sim 15 \mathrm{kDa}$ in length, which is the p.Cys66GlyfsX2 mutant of DAX1 generated by the frameshift 
A
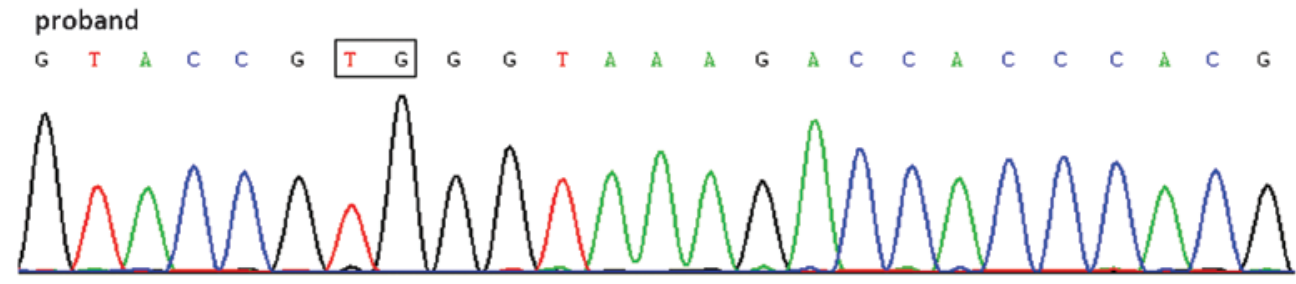

proband's mother
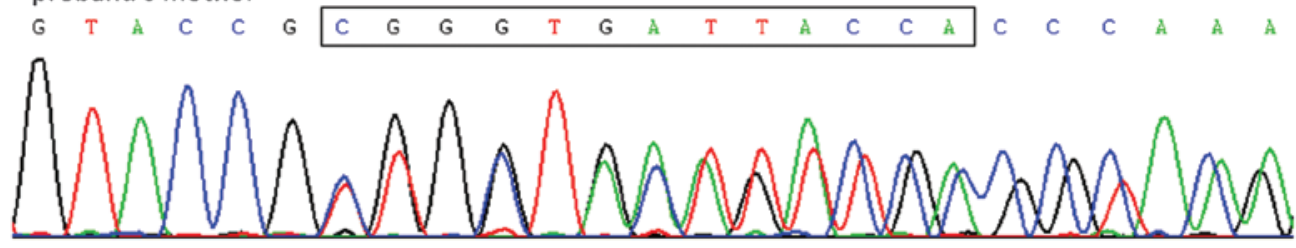

normal control

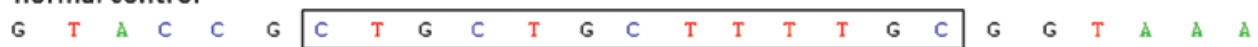

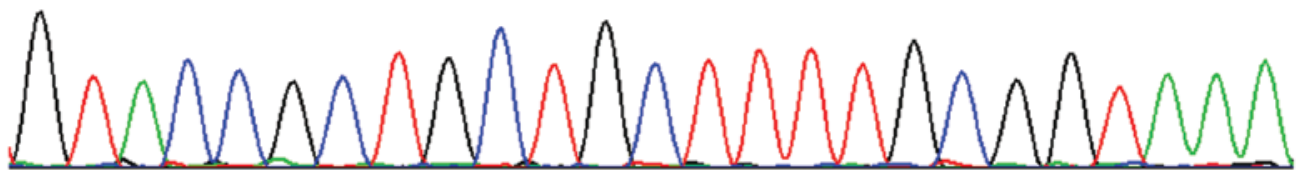

B

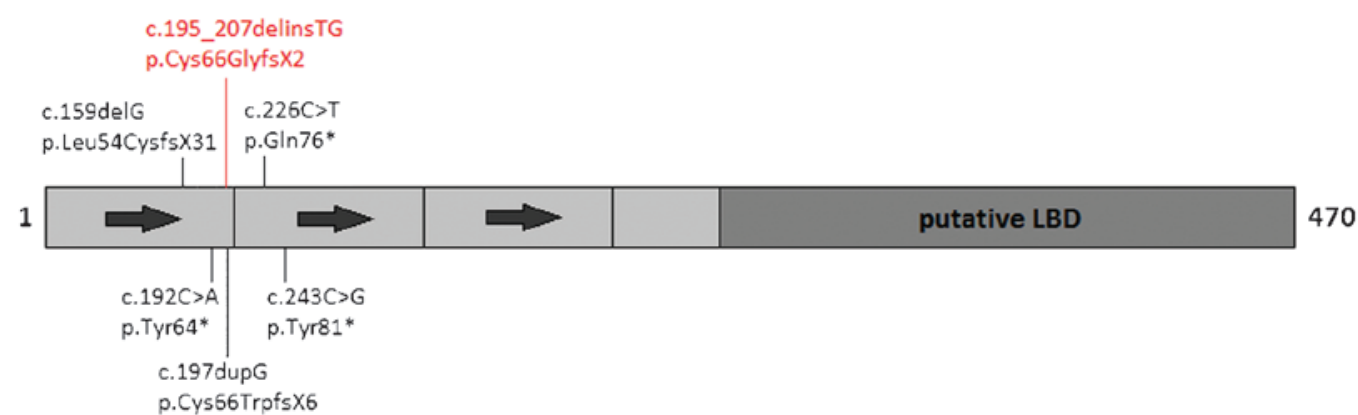

Figure 2. Molecular analysis of the NROB1 gene. (A) Chromatogram of Sanger sequencing showing hemizygous and heterozygous NROB1 c.195_207delinsTG in the proband and the proband's mother, respectively. The corresponding sequences where the indel mutation occurs are indicated in the box. (B) Diagram of the DAX1 protein showing the position of the mutation found in the pedigree (marked in red) compared to the other mutations in adjacent regions described in the Human Gene Mutation Database. LBD, ligand-binding domain.

mutation c.195_207delinsTG in the NROB1 gene. The amount of p.Cys66GlyfsX2 mutant of DAX1 was markedly lower than that of WT DAX1. Furthermore, the p.Cys66GlyfsX2 mutant of DAX1 predominantly located in the cytoplasm. It has been shown that LXXLL motifs and an intact structure of the LBD are crucial for the nuclear localization of DAX1 $(34,35)$. However, the truncated p.Cys66GlyfsX2 mutant of DAX1 only retained the first LXXLL motif, which may have resulted in the abnormal intracellular distribution of the mutant DAX1. Furthermore, this severely truncated DAX1 protein was probably non-functional. Using the C-terminal V5-tag, two additional truncated $\sim 50-\mathrm{kDa}$ proteins that were present in the nucleus as well as in the cytoplasm were detected. Based on a previous in vitro study, an amino-truncated isoform of DAX1, which is normally expressed as a minor protein variant with a partially preserved repressor function, can be generated from an alternate in-frame translation start site (methionine, codon 83) (36). As the alternate translation initiation site is located downstream of the NROBI c.195_207delinsTG

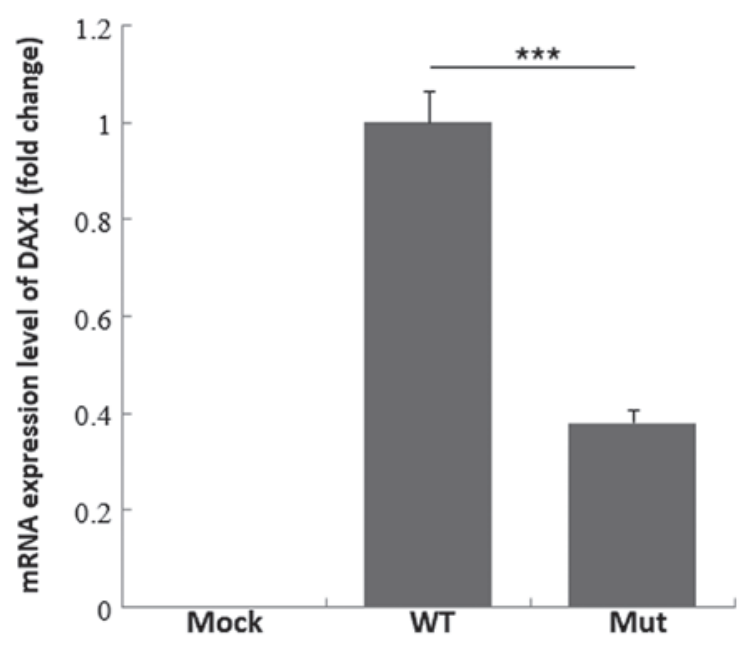

Figure 3. mRNA expression levels of DAX1 in transiently transfected HEK 293T cells. Values are expressed as the mean \pm standard deviation $(\mathrm{n}=3) .{ }^{* * *} \mathrm{P}<0.001$. Mock, empty backbone plasmid; WT, NROB1 wild-type plasmid; Mut, NROB1 c.195_207delinsTG mutant plasmid. 
A

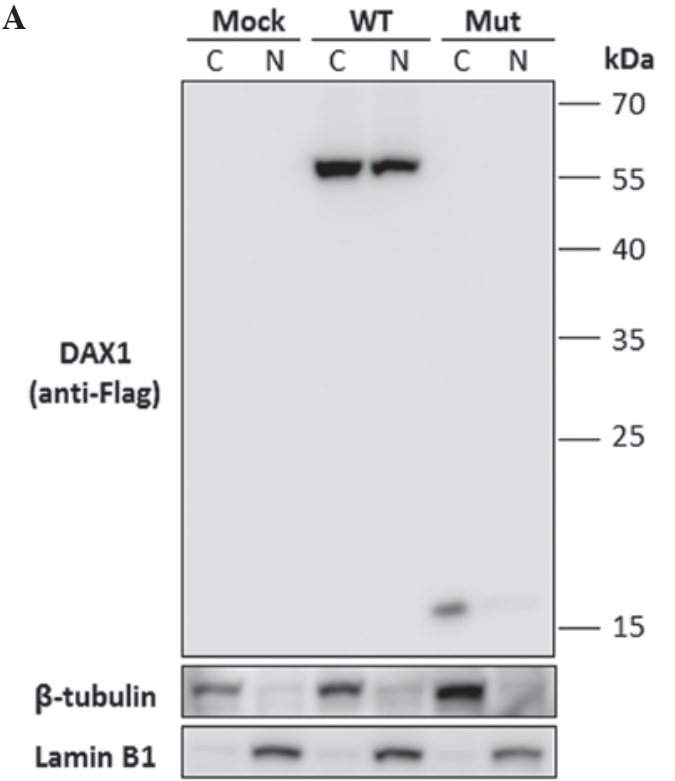

B

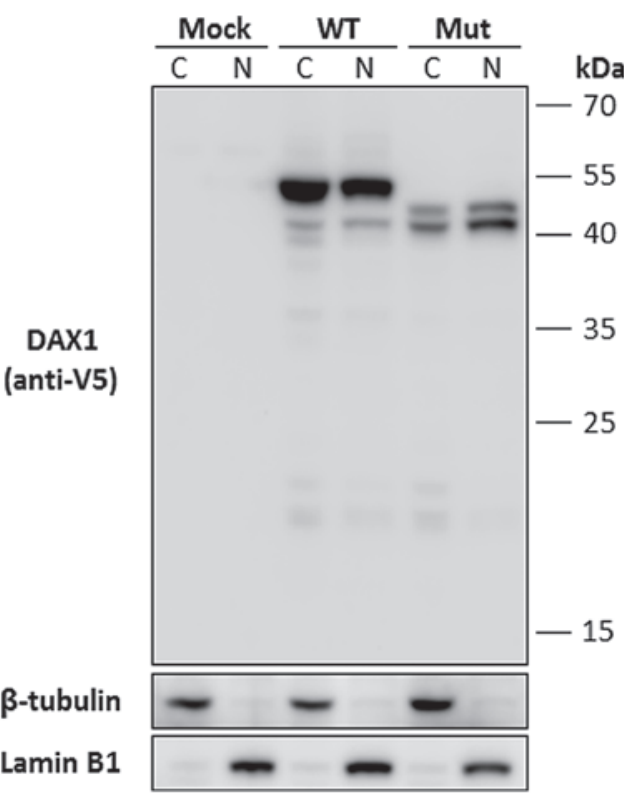

Figure 4. Expression and subcellular localization of DAX1 detected by western blot analysis. (A) N-terminal Flag-tagged NROB1 expression vector was transfected into HEK 293T cells. DAX1 was detected in cytoplasmic and nuclear fractions using an anti-Flag antibody. (B) C-terminal V5-tagged NROBI expression vector was transfected into HEK 293T cells. DAX1 was detected in cytoplasmic and nuclear fractions by using an anti-V5 antibody. $\beta$-tubulin and lamin B1 were used as cytoplasmic and nuclear loading controls, respectively. Mock, empty backbone plasmid; WT, $N R O B 1$ wild-type plasmid; Mut, $N R O B 1$ c.195_207delinsTG mutant plasmid; C, cytoplasm; N, nucleus.

mutation, the formation of this isoform was not affected by the indel mutation. This infers that the lower band, which was also present at low levels in WT DAX1, is the amino-truncated isoform of DAX1. In addition, a higher-molecular weight band was observed in the mutant DAX1 only. Further study is required to determine whether this higher-molecular weight protein resulted from aberrantly spliced mutant mRNA or protein with additional modification induced by the NROBI c.195_207delinsTG mutation.

DAX1 has been shown to be a negative regulator, which acts by repressing the SF-1-mediated transactivation of various

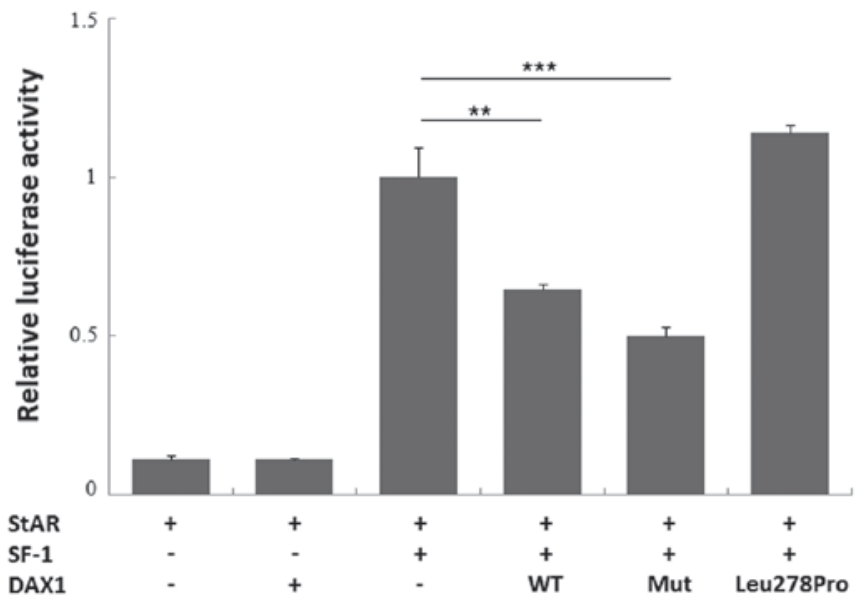

Figure 5. Luciferase assay for SF-1-mediated StAR transactivation. StAR-luc, pRL-SV40, SF-1 and DAX1 (WT, Mut or Leu278Pro) were co-transfected into HEK 293T cells. Luciferase activities were quantified at $24 \mathrm{~h}$ post-transfection and normalized to Renilla luciferase encoded by pRL-SV40. The total amount of DNA was kept constant by complementing with an empty backbone plasmid if necessary. Values are expressed as the mean \pm standard deviation $(\mathrm{n}=3) .{ }^{* *} \mathrm{P}<0.005 ;{ }^{* * *} \mathrm{P}<0.001$. Mut, NROB1 c.195_207delinsTG mutation; WT, NROB1 wild-type plasmid; SF-1, steroidogenic factor 1; StAR, steroidogenic acute regulatory protein.

genes involved in steroidogenesis, such as StAR $(16,17)$. Loss of this inhibitory property in DAX1 through NROB1 mutations was demonstrated to be responsible for the pathology of $\mathrm{X}$-linked AHC and HHG $(3,16,20,23)$. To assess the effects of the c.195_207delinsTG mutation of NROB1 on the repressor function of DAX1, a luciferase assay was performed. The results unexpectedly showed that this mutation did not impair the repressor function of DAX1. Instead, compared to the WT DAX1, the c.195_207delinsTG mutant of DAX1 displayed an even greater ability to suppress SF-1-induced StAR expression. Although the DAX1 Gln37Term and Trp39Term mutations were reported to have a milder phenotype due to the expression of a partially functional, amino-truncated DAX-1 protein, other naturally occurring nonsense or frameshift NROB1 mutations that cause PTC upstream of the putative alternative start site are associated with the classical AHC phenotype (36). These findings suggested that the mutations of NROBI leading to X-linked AHC with $\mathrm{HHG}$ are not uniformly associated with the loss of function or DAX1 and are likely to involve molecular functions other than its role as a repressor of SF-1 activation.

In conclusion, the present study reported on a Chinese pedigree including a case of X-linked AHC and HHG. A novel small indel mutation, c.195_207delinsTG, in the NROB1 gene was identified in the proband. In vitro studies showed that the mutation reduced DAX1 expression and resulted in $\mathrm{N}$-terminal truncated as well as C-terminal truncated DAX1 protein. Furthermore, a Luciferase assay demonstrated that the mutation enhanced the repressor activity of DAX1. The present study indicated the complexity of the underlying mechanisms of the association of NROB1 with X-linked AHC and HHG. Further in vivo studies will help elucidate the biological effects of mutant DAX1 in the development and function of the adrenal gland and hypothalamic-pituitary-gonadal axis. 


\section{Acknowledgements}

The authors would like to thank all participants of the present study.

\section{References}

1. MacMahon HE, Wagner R and Weiner DB: Acute adrenal insufficiency due to congenital defect. Am J Dis Child 94: 282-285, 1957.

2. Zanaria E, Muscatelli F, Bardoni B, Strom TM, Guioli S, Guo W, Lalli E, Moser C, Walker AP, McCabe ER, et al: An unusual member of the nuclear hormone receptor superfamily responsible for X-linked adrenal hypoplasia congenita. Nature 372: 635-641, 1994.

3. Reutens AT, Achermann JC, Ito M, Ito M, Gu WX, Habiby RL, Donohoue PA, Pang S, Hindmarsh PC and Jameson JL: Clinical and functional effects of mutations in the DAX-1 gene in patients with adrenal hypoplasia congenita. J Clin Endocrinol Metab 84 504-511, 1999.

4. Lin L, Gu WX, Ozisik G, To WS, Owen CJ, Jameson JL and Achermann JC: Analysis of DAX1 (NR0B1) and steroidogenic factor-1 (NR5A1) in children and adults with primary adrenal failure: Ten years' experience. J Clin Endocrinol Metab 91: 3048-3054, 2006.

5. Uttley WS: Familial congenital adrenal hypoplasia. Arch Dis Child 43: 724-730, 1968.

6. Habiby RL, Boepple P, Nachtigall L, Sluss PM, CrowleyWF Jr and Jameson JL: Adrenal hypoplasia congenita with hypogonadotropic hypogonadism: Evidence that DAX-1 mutations lead to combined hypothalamic and pituitary defects in gonadotropin production. J Clin Invest 98: 1055-1062, 1996.

7. Zachmann M, Illig R and Prader A: Gonadotropin deficiency and cryptorchidism in three prepubertal brothers with congenital adrenal hypoplasia. J Pediatr 97: 255-257, 1980.

8. Hammond J, Howard NJ, Brookwell R, Purvis-Smith S, Wilcken B and Hoogenraad N: Proposed assignment of loci for X-linked adrenal hypoplasia and glycerol kinase genes. Lancet 1: 54, 1985.

9. Bartley JA, Patil S, Davenport S, Goldstein D and Pickens J: Duchenne muscular dystrophy, glycerol kinase deficiency and adrenal insufficiency associated with Xp21 interstitial deletion. J Pediatr 108: 189-192, 1986.

10. Francke U, Harper JF, Darras BT, Cowan JM, McCabe ER, Kohlschütter A, Seltzer WK, Saito F, Goto J, Harpey JP, et al: Congenital adrenal hypoplasia, myopathy and glycerol kinase deficiency: Molecular genetic evidence for deletions. Am J Hum Genet 40: 212-227, 1987.

11. Muscatelli F, Strom TM, Walker AP, Zanaria E, Récan D, Meindl A, Bardoni B, Guioli S, Zehetner G, Rabl W, et al: Mutations in the DAX-1 gene give rise to both X-linked adrenal hypoplasia congenita and hypogonadotropic hypogonadism. Nature 372: 672-676, 1994.

12. Guo W, Burris TP, Zhang YH, Huang BL, Mason J, Copeland KC, Kupfer SR, Pagon RA and McCabe ER: Genomic sequence of the DAX1 gene: An orphan nuclear receptor responsible for $\mathrm{X}$-linked adrenal hypoplasia congenital and hypogonadotropic hypogonadism. J Clin Endocrinol Metab 81: 2481-2486, 1996.

13. Guo W, Burris TP and McCabe ER: Expression of DAX1, the gene responsible for $\mathrm{X}$-linked adrenal hypoplasia congenita and hypogonadotropic hypogonadism, in the hypothalamic-pituitary-adrenal/gonadal axis. Biochem Mol Med 56 8-13, 1995.

14. Mukai T, Kusaka M, Kawabe K, Goto K, Nawata H, Fujieda K and Morohashi K: Sexually dimorphic expression of Dax-1 in the adrenal cortex. Genes Cells 7: 717-729, 2002

15. Ikeda Y, Swain A, Weber TJ, Hentges KE, Zanaria E, Lalli E, Tamai KT, Sassone-Corsi P, Lovell-Badge R, Camerino G and Parker KL: Steroidogenic factor 1 and Dax-1 colocalize in multiple cell lineages: Potential links in endocrine development. Mol Endocrinol 10: 1261-1272, 1996.

16. Ito M, Yu R and Jameson JL: DAX-1 inhibits SF-1-mediated transactivation via a carboxy-terminal domain that is deleted in adrenal hypoplasia congenital. Mol Cell Biol 17: 1476-1483, 1997.

17. Zazopoulos E, Lalli E, Stocco DM and Sassone-Corsi P: DNA binding and transcriptional repression by DAX-1 blocks steroidogenesis. Nature 390: 311-315, 1997.
18. Suzuki T, Kasahara M, Yoshioka H, Morohashi K and Umesono K: LXXLL-related motifs in Dax-1 have target specificity for the orphan nuclear receptors Ad4BP/SF-1 and LRH-1. Mol Cell Biol 23: 238-249, 2003.

19. Desmangles JC, Lappe JM, Lipaczewski G and Haynatzki G: Accuracy of pubertal Tanner staging self-reporting. J Pediatr Endocrinol Metab 19: 213-221, 2006.

20. Li S and Wilkinson MF: Site-directed mutagenesis: A two-step method using PCR and DpnI. Biotechniques 23: 588-590, 1997.

21. Li N, Liu R, Zhang H, Yang J, Sun S, Zhang M, Liu Y, Lu Y, Wang W, Mu Y, et al: Seven novel DAX1 mutations with loss of function identified in Chinese patients with congenital adrenal hypoplasia. J Clin Endocrinol Metab 95: E104-E111, 2010.

22. Livak KJ and Schmittgen TD: Analysis of relative gene expression data using real-time quantitative PCR and the 2(-Delta Delta C(T)) method. Methods 25: 402-408, 2001.

23. Achermann JC, Ito M, Silverman BL, Habiby RL, Pang S, Rosler A and Jameson JL: Missense mutations cluster within the carboxyl-terminal region of DAX-1 and impair transcriptional repression. J Clin Endocrinol Metab 86: 3171-5, 2001.

24. Peter M, Viemann M, Partsch CJ and Sippell WG: Congenital adrenal hypoplasia: Clinical spectrum, experience with hormonal diagnosis and report on new point mutations of the DAX-1 gene. J Clin Endocrinol Metab 83: 2666-2674, 1998.

25. Esden-Tempska Z, Lewczuk A, Tobias ES, Borozdin W, Kohlhase J and Sworczak K: Delayed diagnosis of adrenal hypoplasia congenita in a patient with a new mutation in the NR0B1 gene. J Pediatr Endocrinol Metab 25: 147-148, 2012.

26. Nakae J, Tajima T, Kusuda S, Kohda N, Okabe T, Shinohara N, Kato M, Murashita M, Mukai T, Imanaka $K$ and Fujieda $K$ : Truncation at the C-terminus of the DAX-1 protein impairs its biological actions in patients with X-linked adrenal hypoplasia congenita. J Clin Endocrinol Metab 81: 3680-3685, 1996.

27. Tsai WY and Tung YC: Novel deletion mutations of the DAX1 (NR0B1) gene in two Taiwanese families with X-linked adrenal hypoplasia congenita. J Pediatr Endocrinol Metab 18: 991-997, 2005.

28. Domenice S, Latronico AC, Brito VN, Arnhold IJ, Kok F and Mendonca BB: Adrenocorticotropin-dependent precocious puberty of testicular origin in a boy with X-linked adrenal hypoplasia congenita due to a novel mutation in the DAX1 gene. J Clin Endocrinol Metab 86: 4068-4071, 2001.

29. Wheeler B, George PM, Mackenzie K, Hunt P, Potter HC and Florkowski CM: Three cases of congenital adrenal hypoplasia with novel mutations in the (NROB1) DAX-1 gene. Ann Clin Biochem 45: 606-609, 2008.

30. García-Malpartida K, Gómez-Balaguer M, Solá-Izquierdo E, Fuentes-Pardilla MJ, Jover-Fernández A, Sanz-Ruiz I and Hernández-Mijares A: A novel mutation in DAX1 (NR0B1) causing X-linked adrenal hypoplasia congenita: Clinical, hormonal and genetic analysis. Endocrine 36: 275-280, 2009.

31. Zhang YH, Huang BL, Anyane-Yeboa K, Carvalho JA, Clemons RD, Cole T, De Figueiredo BC, Lubinsky M, Metzger DL, Quadrelli R, et al: Nine novel mutations in NR0B1 (DAX1) causing adrenal hypoplasia congenita. Hum Mutat 18: $547,2001$.

32. Brogna $\mathrm{S}$ and Wen J: Nonsense-mediated mRNA decay (NMD) mechanisms. Nat Struct Mol Biol 16: 107-113, 2009.

33. Lalli E, Ohe K, Hindelang C and Sassone-Corsi P: Orphan receptor DAX-1 is a shuttling RNA binding protein associated with polyribosomes via mRNA. Mol Cell Biol 20: 4910-4921, 2000.

34. Kawajiri K, Ikuta T, Suzuki T, Kusaka M, Muramatsu M, Fujieda K, Tachibana M and Morohashi K: Role of the LXXLL-motif and activation function 2 domain in subcellular localization of Dax-1 (dosage-sensitive sex reversal-adrenal hypoplasia congenita critical region on the $\mathrm{X}$ chromosome, gene 1). Mol Endocrinol 17: 994-1004, 2003.

35. Lehmann SG, Lalli E and Sassone-Corsi P: X-linked adrenal hypoplasia congenita is caused by abnormal nuclear localization of the DAX-1 protein. Proc Natl Acad Sci USA 99: 8225-8230, 2002.

36. Ozisik G, Mantovani G, Achermann JC, Persani L, Spada A, Weiss J, Beck-Peccoz P and Jameson JL: An alternate translation initiation site circumvents an amino-terminal DAX1 nonsense mutation leading to a mild form of X-linked adrenal hypoplasia congenital. J Clin Endocrinol Metab 88: 417-423, 2003. 